\title{
The spectroscopic orbit of Capella revisited ${ }^{\star} \star \star$
}

\author{
M. Weber and K. G. Strassmeier
}

\author{
Leibniz-Institut für Astrophysik Potsdam (AIP), An der Sternwarte 16, 14482 Potsdam, Germany \\ e-mail: [MWeber,KStrassmeier]@aip.de
}

Received 14 March 2011 / Accepted 9 April 2011

\begin{abstract}
Context. Capella is among the few binary stars with two evolved giant components. The hotter component is a chromospherically active star within the Hertzsprung gap, while the cooler star is possibly helium-core burning.

Aims. The known inclination of the orbital plane from astrometry in combination with precise radial velocities will allow very accurate masses to be determined for the individual Capella stars. This will constrain their evolutionary stage and possibly the role of the active star's magnetic field on the dynamical evolution of the binary system.

Methods. We obtained a total of 438 high-resolution échelle spectra during the years 2007-2010 and used the measured velocities to recompute the orbital elements. Our double-lined orbital solution yields average residuals of $64 \mathrm{~m} \mathrm{~s}^{-1}$ for the cool component and $297 \mathrm{~m} \mathrm{~s}^{-1}$ for the more rapidly rotating hotter component.

Results. The semi-amplitude of the cool component is smaller by $0.045 \mathrm{~km} \mathrm{~s}^{-1}$ than the orbit determination of Torres et al. from data taken during 1996-1999 but more precise by a factor of 5.5, while for the hotter component it is larger by $0.580 \mathrm{~km} \mathrm{~s}^{-1}$ and more precise by a factor of 3.6. This corresponds to masses of $2.573 \pm 0.009 M_{\odot}$ and $2.488 \pm 0.008 M_{\odot}$ for the cool and hot component, respectively. Their relative errors of $0.34 \%$ and $0.30 \%$ are about half of the values given in Torres et al. for a combined literaturedata solution but with absolute values different by $4 \%$ and $2 \%$ for the two components, respectively. The mass ratio of the system is therefore $q=M_{\mathrm{A}} / M_{\mathrm{B}}=0.9673 \pm 0.0020$.

Conclusions. Our orbit is the most precise and also likely to be the most accurate ever obtained for Capella.
\end{abstract}

Key words. stars: individual: Capella ( $\alpha$ Aur) - starspots - techniques: radial velocities - binaries: spectroscopic - stars: late-type

\section{Introduction}

The spectrum of the Capella ( $\alpha$ Aurigae, $V=0.07$ mag) binary has been studied for over a century and has been observed at practically all wavelengths from X-rays to the radio. The literature is too numerous to be cited here, but Torres et al. (2009) contains references to almost all of the significant work, in particular those relevant to the present paper. The studies of Barlow et al. (1993) and Hummel et al. (1994) of all aspects of the astrometric and spectroscopic orbits are cited here to emphasize their historical importance.

Capella is one of the few binary systems with two moderately evolved giants, most often cited as G8 III for the primary and G0-1 III for the secondary. Atypical of RS CVn binaries, the hotter component of Capella appears to be the more active star rather than the cooler, usually rapidly rotating component. This is unsurprising for its spectral type and evolutionary status as this places the hot component in the Hertzsprung gap where rapid redistribution of the internal angular momentum is taking place, eventually followed by dredge up of high-angular momentum material even to the surface. Our overall goal is to learn more about this component by mapping its surface spots. For comparison, we previously investigated a very similar but single G0 III star (31 Com; Strassmeier et al. 2010a). Quite unexpectedly, we found a large polar spot in Doppler images despite

$\star$ Based on data obtained with the STELLA robotic telescope in Tenerife, an AIP facility jointly operated by AIP and IAC.

$\star \star$ Full Table 1 is only available at the CDS via anonymous ftp to cdsarc.u-strasbg.fr $(130.79 .128 .5)$ or via

http://cdsarc.u-strasbg.fr/viz-bin/qcat?]/A+A/531/A89 the predicted depth of its convective envelope of less than $20 \%$ being much too small to account for flux-tube deflection towards the rotational poles. The star's rotation period was found to be $6.80 \pm 0.06$ days from MOST data. Radial-velocity variations with a full amplitude of $270 \mathrm{~m} \mathrm{~s}^{-1}$ and a period of $6.76 \mathrm{~d}$ were detected from our STELLA spectra, which we also interpreted as being caused by the stellar rotation and made us confident in the use of STELLA for this high-precision work. The stellar mass and age for $31 \mathrm{Com}$ were determined to be $2.6 \pm 0.1 M_{\odot}$ and $\approx 540$ Myr from a comparison with evolutionary tracks. To first order, we would expect similar values for the hot secondary component of Capella.

In this paper, we report on our ongoing monitoring of Capella and present a new improved orbital solution. The secondary's comparably featureless spectrum with respect to the cooler component and its large rotational line broadening of $35 \mathrm{~km} \mathrm{~s}^{-1}$ make its observation difficult in spite of the favorable light ratio cool/hot component of $\approx 0.9$ at visual wavelengths. Our own earlier work on Capella was based on either snapshot spectroscopy (Strassmeier \& Fekel 1990) or H $\alpha$ photometry (Strassmeier et al. 2001). The latter paper presented the detection of a $106 \pm 3 \mathrm{~d}$ photometric period for the late-G giant, which we interpreted to be its rotation period synchronized to the orbital motion. A period of $8.64 \pm 0.09 \mathrm{~d}$ was found for the $\mathrm{G} 0-1 \mathrm{gi}-$ ant, although it is less well defined and certainly less accurate than the formal error suggests but in agreement with an independent determination from the He I equivalent widths by Katsova \& Scherbakov (1998). However, the knowledge of accurate absolute dimensions, in particular the component masses, radii, and rotational periods, are essential for comparing a system's angular 
momentum evolution with model predictions. Its present location in the Hertzsprung gap suggests that there are rapid changes in its internal structure with a deepening convection zone and associated changes in the total stellar moment of inertia (e.g. Kim $\&$ Barnes 2008). Deep mixing processes at this stage are also predicted to have a direct impact on the visible surface rotation and chemical abundance (Böhm-Vitense 2004). Precise astrophysical parameters of stars in this evolutionary stage may thus help us to understand the angular-momentum loss in late-type stars.

We employed our new robotic spectroscopic facility STELLA in Tenerife, Canary Islands to continuously monitor Capella for a total of nearly four consecutive years with a cadence of one spectrum for every useable night. The instrument and the data are described in Sect. 2. A new SB2 orbit with, so far, unprecedented accuracy is presented in Sect. 3. We discuss its details in Sect. 4. In a forthcoming paper, we present the first attempt to derive a Doppler image of the surface of the rapidly rotating secondary component.

\section{STELLA/SES spectroscopy in 2007-2010}

High-resolution time-series spectroscopy was obtained with the STELLA Échelle Spectrograph (SES) at the robotic 1.2-m STELLA-I telescope in Tenerife, Spain (Strassmeier et al. 2010b). A total of 438 échelle spectra were acquired over the course of $31 / 2$ years $(1312 \mathrm{~d})$. The SES is a fiber-fed whitepupil échelle spectrograph with a fixed wavelength format of 388-882 nm. Despite increasing inter-order gaps in the red, it records the range $390-720 \mathrm{~nm}$ continuously. Its two-pixel resolution is $R=55000$. The CCD is currently an E2V 42-40 $2048 \times 204813.5 \mu$ m-pixel device.

Integrations on Capella were set to exposure times of $15 \mathrm{~s}$ and $60 \mathrm{~s}$ (until end of 2007) and achieved signal-to-noise ratios $(\mathrm{S} / \mathrm{N})$ in the range 200-500: 1 per resolution element, depending on weather conditions. The spectra were obtained between July 2007 (JD 2454305.7 ) and March 2011. Just 14 primary and 7 secondary measurements were discarded because their data were of too low $\mathrm{S} / \mathrm{N}$ due to clouds. Our data are automatically reduced and extracted with the IRAF-based STELLA data-reduction pipeline (see Weber et al. 2008). The two-dimensional (2d) images were corrected for bad pixels and cosmic rays. Bias levels were removed by subtracting the average overscan from each image followed by the subtraction of the mean of the (already overscan-subtracted) master bias frame. The target spectra were flattened by dividing by the master flat, which was normalized to unity. The robot's time series also includes nightly and daily Th-Ar comparison-lamp exposures for wavelength calibration and spectrograph focus monitoring. A continuous monitoring of the environmental parameters inside and outside the spectrograph room, most notably of temperature and barometric pressure, allows us to apply proper corrections. For details of the échelle data reduction with particular emphasis on the temperature and pressure dependences of the SES, we refer to Weber et al. (2008).

Twenty-two radial velocity standard stars were observed with the same set-up and analyzed in Strassmeier et al. (2010c, 2011). The STELLA system appears to have a zero-point offset with respect to CORAVEL (Udry et al. 1999) of $0.50 \pm$ $0.21 \mathrm{~km} \mathrm{~s}^{-1}$, and a comparable offset of $0.46 \pm 0.64 \mathrm{~km} \mathrm{~s}^{-1}$ with respect to the 17 stars in common with Scarfe (2010). The highest external rms radial-velocity precision over the four years of observation was around $30 \mathrm{~m} \mathrm{~s}^{-1}$ for late-type stars with narrow spectral lines. All velocities in this paper are on the STELLA
Table 1. Barycentric STELLA radial velocities of Capella.

\begin{tabular}{lllll}
\hline \hline HJD & $v_{\mathrm{A}}$ & \multicolumn{2}{c}{$\sigma_{\mathrm{A}}$} & $\begin{array}{c}v_{\mathrm{B}} \\
\left(\mathrm{km} \mathrm{s}^{-1}\right)\end{array}$ \\
& \multicolumn{4}{c}{$\sigma_{\mathrm{B}}$} \\
\hline 2454305.724 & 44.932 & 0.029 & 14.209 & 0.381 \\
2454308.712 & 40.642 & 0.038 & 18.032 & 0.455 \\
$\ldots$ & $\ldots$ & $\ldots$ & $\ldots$ & $\ldots$ \\
2455616.490 & 30.384 & 0.034 & 29.515 & 0.222 \\
2455617.482 & 31.949 & 0.030 & 28.070 & 0.254 \\
\hline
\end{tabular}

Notes. Full version available at the CDS.

zero-point scale. The individual velocities are listed in Table 1, available only in electronic form via CDS Strasbourg.

\section{New spectroscopic orbital elements}

\subsection{High-precision radial velocities}

During the initial year of STELLA operations, the external rms values were significantly larger $\left(120 \mathrm{~m} \mathrm{~s}^{-1}\right)$ than thereafter $\left(30 \mathrm{~m} \mathrm{~s}^{-1}\right)$ and included a radial velocity offset of about $300 \mathrm{~m} \mathrm{~s}^{-1}$. The final radial velocities are barycentric and corrected for Earth's rotation.

The STELLA velocities listed here are determined from a simultaneous cross-correlation of 62 échelle orders with a proper synthetic spectrum. To perform this, we selected from a precomputed grid of synthetic spectra from ATLAS-9 atmospheres (Kurucz 1993) two spectra that match the respective target spectral classifications. For Capella A, we chose the $T_{\text {eff }}=5000 \mathrm{~K}$ model, and for Capella B the $T_{\text {eff }}=5500 \mathrm{~K}$ model, both with $\log g=3.0$ and solar metallicity. We combined the two template spectra to one artificial spectrum using $v \sin i$ of $4.5 \mathrm{~km} \mathrm{~s}^{-1}$ and $35 \mathrm{~km} \mathrm{~s}^{-1}$ for the $\mathrm{A}$ and $\mathrm{B}$ component, respectively, and a macroturbulence of $5 \mathrm{~km} \mathrm{~s}^{-1}$. We adjusted the (wavelength dependent) intensity ratio using G8 III and G0 III flux-calibrated spectra from Pickels (1998) multiplied with 0.955. We then applied a series of radial velocity differences between the two components, and computed a two-dimensional cross-correlation function for each of these shifts. The highest correlation value in the resulting two-dimensional image corresponds to the measured velocity of component $\mathrm{A}$ in one dimension, and the velocity difference of the two components in the other dimension. To estimate the uncertainties in this method, we evaluated a series of 1000 Monte-Carlo variations in these two-dimensional images using only $63 \%$ of the original number of orders, estimating $\sigma$ to be $1 / 1.349$ of the interquartile range of the resulting distribution.

To correct for systematic radial velocity offsets induced by the measuring technique, we created simulated stellar spectra from the above templates at exactly the same wavelength spacing as the original observations and corrected the measured radial velocities with these offsets. We initially performed our analysis with 19 échelle orders but the final analysis was performed with all usable orders, which turned out to be 62 out of 82 in our case. Figure 1 illustrates the systematic errors and their dependance on the number of orders, 19 compared to 62, and the wavelength range used. Doing the analysis with just 19 orders resulted in a $K_{\mathrm{B}}$ of about $400 \mathrm{~m} \mathrm{~s}^{-1}$ smaller than with 62 orders, even when taking the corrections from Fig. 1 into account. This shows that the systematic effects are not fully taken into account by the simulations.

We also correct the radial velocities of the B component for the difference in gravitational redshift according to 


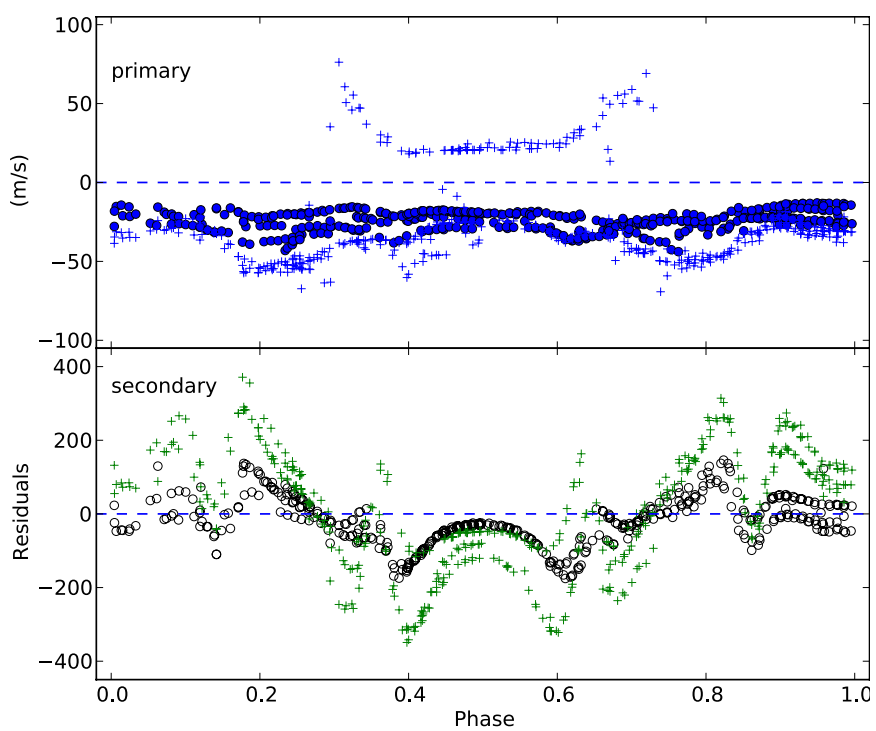

Fig. 1. Residual velocities from simulated spectra. Circles denote the final setup using 62 échelle orders. Pluses indicate the residuals when using only 19 orders. A simulated spectrum was computed for the time of every observed spectrum, and the same method as used for the observations was applied to derive the radial velocities of the two components. The offsets shown here are the differences between the velocities used to construct the simulated spectra and the values measured. Systematic offsets mostly affect the secondary star, with absolute values peaking at about $50 \%$ of the single measurement error of typically $300 \mathrm{~m} \mathrm{~s}^{-1}$ for component B. Increasing the number of orders and thus the wavelength range clearly suppresses the systematic errors.

Lindgren \& Dravins (2003). For component A, this amounts to $132 \mathrm{~m} \mathrm{~s}^{-1}$ and for component $\mathrm{B}$ to $177 \mathrm{~m} \mathrm{~s}^{-1}$ with the masses and radii from Table 2, i.e. a net differential offset of $45 \mathrm{~m} \mathrm{~s}^{-1}$. After applying this correction, a residual offset of the respective center-of-mass velocities of the two components of $59 \mathrm{~m} \mathrm{~s}^{-1}$ remained. This small offset could be caused by the different lineshapes of the two stars, e.g. produced by either convective blueor redshifts (see e.g. Gray 2005). Therefore, we applied a total correction of $104 \mathrm{~m} \mathrm{~s}^{-1}$ to the radial velocities of the B component. The main effect of this correction is a small decrease in the secondary's velocity amplitude. We note that all of above corrections still amount to changes in the final parameters of smaller than $1 \sigma$.

\subsection{Spectroscopic orbit}

Figure 2 presents our new STELLA velocities and compares them with the computed velocity curves.

We solved for the usual elements of a double-lined spectroscopic binary using the general least-squares fitting algorithm MPFIT (Markwardt 2009), utilizing the reciprocal values of $\sigma$ from Table 1 as weights. For solutions with non-zero eccentricity, we used the prescription of Danby \& Burkardt (1983) to calculate the eccentric anomaly. The orbital period was fixed at $104.02173 \mathrm{~d}$ (Torres et al. 2009). We initially assumed a zero eccentricity, but the final corrected data yields a best-fit solution at $e=0.00087 \pm 0.00013$ and $\omega=345 \pm 9.7$ for both the SB2 solution and the solution of the primary's velocities alone. Using only the secondary's velocities, we obtain a zero eccentricity. The rms of the eccentric solution is $64 \mathrm{~m} \mathrm{~s}^{-1}$ and $297 \mathrm{~m} \mathrm{~s}^{-1}$ for the primary and secondary velocities, respectively, which is slightly better than the $65 \mathrm{~m} \mathrm{~s}^{-1}$ and $301 \mathrm{~m} \mathrm{~s}^{-1}$ obtained
Table 2. Spectroscopic orbital elements of Capella.

\begin{tabular}{|c|c|c|}
\hline Parameter & Our value & Torres et al. \\
\hline$P$ (days) & & $104.02173 \pm 0.00022$ \\
\hline$T_{\text {Periastron }}$ (HJD 24+) & $54389 \pm 2.8$ & $47528.514 \pm 0.016$ \\
\hline$\gamma\left(\mathrm{km} \mathrm{s}^{-1}\right)$ & $29.9378 \pm 0.0026$ & $29.653 \pm 0.035$ \\
\hline$K_{\mathrm{A}}\left(\mathrm{km} \mathrm{s}^{-1}\right)$ & $25.960 \pm 0.0065$ & $26.005 \pm 0.036$ \\
\hline$K_{\mathrm{B}}\left(\mathrm{km} \mathrm{s}^{-1}\right)$ & $26.840 \pm 0.024$ & $26.260 \pm 0.087$ \\
\hline$e$ & $0.00087 \pm 0.00013$ & 0.0 \\
\hline$\omega(\mathrm{deg})$ & $345 \pm 9.7$ & - \\
\hline$a_{\mathrm{A}} \sin i\left(10^{6} \mathrm{~km}\right)$ & $37.134 \pm 0.0093$ & \\
\hline$a_{\mathrm{B}} \sin i\left(10^{6} \mathrm{~km}\right)$ & $38.392 \pm 0.034$ & \\
\hline$M_{\mathrm{A}} \sin ^{3} i\left(M_{\odot}\right)$ & $0.8064 \pm 0.0014$ & \\
\hline$M_{\mathrm{B}} \sin ^{3} i\left(M_{\odot}\right)$ & $0.7800 \pm 0.0008$ & \\
\hline$N_{\mathrm{A}}, N_{\mathrm{B}}{ }^{a}$ & 424,431 & $504,162^{b}$ \\
\hline $\mathrm{rms}_{\mathrm{A}}\left(\mathrm{m} \mathrm{s}^{-1}\right)$ & 64 & \\
\hline $\mathrm{rms}_{\mathrm{B}}\left(\mathrm{m} \mathrm{s}^{-1}\right)$ & 297 & \\
\hline $\begin{array}{l}\text { mass ratio, } q \equiv M_{\mathrm{B}} / M_{\mathrm{A}} \\
\text { inclination, } i(\mathrm{deg})\end{array}$ & $0.9673 \pm 0.0020$ & $\begin{array}{l}0.9903 \pm 0.0036 \\
137.21 \pm 0.05\end{array}$ \\
\hline$M_{\mathrm{A}}\left(M_{\odot}\right)$ & $2.573 \pm 0.009$ & $2.466 \pm 0.018$ \\
\hline$M_{\mathrm{B}}\left(M_{\odot}\right)$ & $2.488 \pm 0.008$ & $2.443 \pm 0.013$ \\
\hline$R_{\mathrm{A}}\left(R_{\odot}\right)$ & & 11.87 \\
\hline$R_{\mathrm{B}}\left(R_{\odot}\right)$ & & 8.75 \\
\hline
\end{tabular}

Notes. ${ }^{(a)} N$ is the number of measurements for each component. (b) A total number of 1015 measurements were used including astrometry.

from the circular solution. The eccentricity is also consistent with the values of $e=0.00083 \pm 0.00005$ and $\omega=334.8 \pm 4.7$ that Torres et al. (2009) derived from the astrometric measurements of Hummel et al. (1994), and practically identical to their result when all radial-velocity and astrometric data were combined $(0.00087 \pm 0.00021$ and $324 \pm 14)$.

\section{Discussion and conclusions}

Most prior studies of the spectroscopic orbit of Capella have found that the early $\mathrm{G}$ star is the least massive component in the Capella system. The only exception is an early orbit by Struve \& Kung (1953) from McDonald-Observatory spectra, as noted by Barlow et al. (1993). Torres et al. (2009) collected most previously obtained spectroscopic orbits and documented the decrease in the measured semi-amplitude of the secondary with ever increased observational precision. The difficulty in measuring the radial velocity of the secondary (of a zero magnitude star!) is thereby nicely demonstrated. The earliest orbital solutions had a secondary to primary mass ratio of $\approx 0.74$, which became $\approx 0.87$ by the mid eighties in the past century, $\approx 0.94$ in the nineties, and $0.9903 \pm 0.0036$ in the study of Torres et al. (2009). In the present paper, we verify the almost unity mass ratio but confine it to $0.9673 \pm 0.0020$ and derive masses good to $0.3 \%$.

The two previously highest quality data sets - Torres et al. (2009) from CfA observations, and Barlow et al. (1993) from a combination of DAO, McDonald, and KPNO observations have comparable rms for the primary of 0.46 and $0.47 \mathrm{~km} \mathrm{~s}^{-1}$ but 0.91 and $1.38 \mathrm{~km} \mathrm{~s}^{-1}$ for the secondary, respectively. The CfA data set appears to be significantly more precise for the secondary velocities than the combined Barlow et al. (1993) velocities. However, the CfA velocities are not free of systematics. Firstly, they were obtained from single-order échelle spectra with a wavelength coverage of just $45 \AA$, 117 times smaller than our STELLA/SES data. As shown by the authors themselves, 

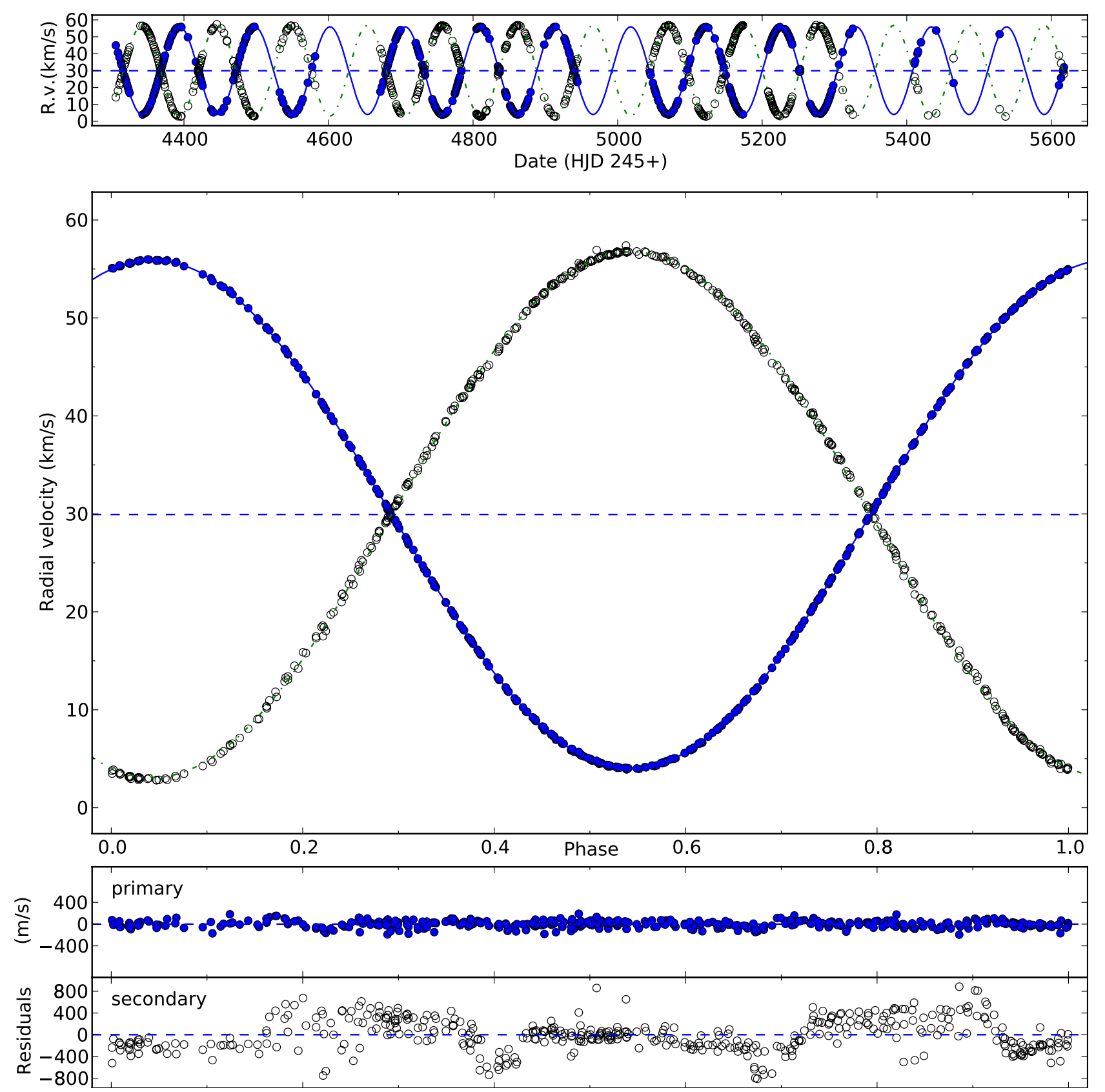

Fig. 2. The STELLA radial velocities compared with our newly computed orbit. Filled circles denote the cool component, star A, open circles the hot component, star B. The two top panels show the radial velocities versus HJD and versus orbital phase, the two bottom panels show the residuals versus phase for the primary and the secondary, respectively. The lines are computed from our elements in Table 2 . The horizontal dashed line in the middle panel is the systemic velocity. The residuals show a phase-dependent behavior in both components, but the overall $\mathrm{rms}$ of $64 \mathrm{~m} \mathrm{~s}^{-1}$ and $297 \mathrm{~m} \mathrm{~s}^{-1}$ for the two components fit the uncertainties of the individual measurements quite well.

this introduces phase-dependent radial velocity variations of the secondary star of up to $\pm 1 \mathrm{~km} \mathrm{~s}^{-1}$, which are produced by residual blending and spectral lines moving in and out of the crosscorrelation window. All CfA velocities had to be corrected for this effect before further use. Secondly, when the CfA velocities from both components were solved for separately, their best solution was found for $\gamma$-velocities that differ by $0.27 \pm$ $0.08 \mathrm{~km} \mathrm{~s}^{-1}$, with the B-component value being the smaller one. This is not uncommon in double-lined solutions but this difference also persisted in their global solution. Spot activity on both stars can account for such an effect but should statistically cancel out for a long enough database timeline unless phenomena such as active spot longitudes or variable spot lifetimes play a role.
Our STELLA data are not affected as strongly by the above problems, the precision of an individual measurement even when compared to the best previous data being already higher by a factor 3-4, owing to spectrograph stability and higher spectral resolution. Our rms from the orbital solution is $64 \mathrm{~m} \mathrm{~s}^{-1}$ for the primary and $297 \mathrm{~m} \mathrm{~s}^{-1}$ for the secondary. The sampling is also unprecedented and a total of 424 velocities for the primary and 431 for the secondary were available for our analysis. If we use the best-fit orbital period from the STELLA data alone, i.e. $104.0243 \pm 0.0007 \mathrm{~d}$, we find a practically indistinguishable orbit from that listed in Table 2 with rms-values of within $1 \mathrm{~m} \mathrm{~s}^{-1}$ and $4 \mathrm{~m} \mathrm{~s}^{-1}$ for the cool and hot components, respectively. Because our time coverage is shorter than that considered in the 
combined Torres et al. orbit, $1312 \mathrm{~d}$ versus $5037 \mathrm{~d}$, we chose to adopt their orbital period from their combined solution. Taking our velocity offset of $+0.503 \mathrm{~km} \mathrm{~s}^{-1}$ with respect to CORAVEL into account, our $\gamma$ velocity is smaller by $0.218 \mathrm{~km} \mathrm{~s}^{-1}$ than the Torres et al. value of $29.653 \pm 0.053 \mathrm{~km} \mathrm{~s}^{-1}$ but, more importantly, more precise by a factor 20 . The semi-amplitude of component A from STELLA is smaller by just $0.045 \mathrm{~km} \mathrm{~s}^{-1}$ but more precise by a factor of 5.5, while for component B it is larger by $0.580 \mathrm{~km} \mathrm{~s}^{-1}$ and more precise by a factor of 3.6. As noted in Sect. 3.1, we obtain a $K_{\mathrm{B}}$ value within the error bars similar to the one found by Torres et al. when using only 19 spectral orders. We therefore conclude that the systematic errors in the secondary velocity make $K_{\mathrm{B}}$ appear smaller (see Fig. 1). Because of the above rms numbers and that an inspection of the radial-velocity curves in Fig. 2 shows residual systematic errors only at the level of the accuracy of a single measurement, we conclude that our orbit is also more accurate. We speculate that the inclusion of the many different data sets in the Torres et al. solution, spectroscopic as well as astrometric, and their simultaneous global approach with a total of 26 free adjustable parameters creates high precision but lesser than the hoped for accuracy, possibly because of remaining systematics in the individual data sets.

The mass ratio of a binary with two evolved giant components is of critical importance for the determination of their evolutionary status. This is particularly obvious for the Capella system as we have the case where two practically equally massive giants occupy rather different locations in the H-R diagram, and yet have the same age. Mostly by virtue of its rotational line broadening, the hotter B component is the one with the more uncertain parameters. Its spectral classification, however, unambiguously places it within the Hertzsprung gap where rapid changes in terms of surface chemistry, angular momentum etc. are taking place. The orbital elements from Table 2, combined with the inclination of the orbital plane from the astrometric orbit of $137.21^{\circ}$ (again cf. Torres et al.), give masses of $2.573 \pm$ $0.009 M_{\odot}$ and $2.488 \pm 0.008 M_{\odot}$ for the A and B components, respectively. These are masses with relative errors of $0.34 \%$ and $0.30 \%$, about half of the errors given in Torres et al., and with absolute values that differ by $4 \%$ and $2 \%$ for the two stars. We note that we continue to denote the A component as the primary because it is the dominant source in the combined spectrum but also recall that the secondary is the brighter of the two components at optical wavelengths (Griffin \& Griffin 1986; Strassmeier \& Fekel 1990).

Acknowledgements. STELLA was made possible by funding through the State of Brandenburg (MWFK) and the German Federal Ministry of Education and Research (BMBF). The facility is a collaboration of the AIP in Brandenburg with the IAC in Tenerife. We thank all engineers and technicians involved, in particular Manfred Woche and Emil Popow and his team as well as Ignacio del Rosario and Miquel Serra from the IAC Tenerife day-time crew. We thank the referee, Frank Fekel, for his constructive critisism.

\section{References}

Barlow, D. J., Fekel, F. C., \& Scarfe, C. D. 1993, PASP, 105, 476 Böhm-Vitense, E. 2004, AJ, 128, 2435

Danby, J. M. A., \& Burkardt, T. M. 1983, Celest. Mech., 31, 95

Gray, D. F. 2005, PASP, 117, 711

Griffin, R., \& Griffin, R. 1986, J. Astrophy. Astr., 7, 45

Hummel, C. A., Armstrong, J. T., Quirrenbach, A., et al. 1994, AJ, 107, 1859

Katsova, M. M., \& Scherbakov, A. G. 1998, Astron. Rep., 42, 485

Kim, Y.-C., \& Barnes, S. A. 2008, in The Art of Modeling Stars in the 21st Century, ed. L. Deng, \& K. L. Chan (Cambridge: Cambridge Univ. Press), IAU Symp., 252, 117

Kurucz, R. L. 1993, ATLAS-9, CD-ROM \#13

Lindegren, L., \& Dravins, D. 2003, A\&A, 401, 1185

Markwardt, C. B. 2009, in Astronomical Data Analysis Software and Systems XVIII, ed. D. A. Bohlender, D. Durand, \& P. Dowler, ASPC Ser. 411,251

Pickles, A. J. 1998, PASP, 110, 863

Scarfe, C. D. 2010, Obs, 130, 214

Strassmeier, K. G., \& Fekel, F. C. 1990, A\&A, 230, 389

Strassmeier, K. G., Reegen, P., \& Granzer, T. 2001, AN, 322, 115

Strassmeier, K. G., Granzer, T., Kopf, M., et al. 2010a, A\&A, 520, A52

Strassmeier, K. G., Granzer, T., Weber, M., et al. 2010b, Adv. in Astr., 19

Strassmeier, K. G., Weber, M., Granzer, T., \& Dall, T. H. 2010c, AN, 331, 368

Strassmeier, K. G., Weber, M., Granzer, T., \& Järvinen, S. 2011, AN, submitted Struve, O., \& Kung, S. M. 1953, ApJ, 117, 1

Torres, G., Claret, A., \& Young, P. A. 2009, ApJ, 700, 1349

Udry, S., Mayor, M., Maurice, E., et al. 1999, in Precise Stellar Radial Velocities, IAU Coll., 170, ed. J. B. Hearnshaw, \& C. D. Scarfe, ASPC Ser., 185, 383

Weber, M., Granzer, T., Strassmeier, K. G., \& Woche, M. 2008, Proc. SPIE, 7019, 70190L 